\title{
MENTAL FATIGUE AS A CONDITIONER OF THE TACTICAL AND PHYSICAL RESPONSE IN SOCCER PLAYERS: A PILOT STUDY
}

original paper

(1) University School of Physical Education in Wroclaw

DOI: https://doi.org/10.5114/hm.2018.76075

\author{
CAITO ANDRE KUNRATH ${ }^{1,2}$, FELIPPE CARDOSO ${ }^{1,2}$, FABIO YUZO NAKAMURA ${ }^{3,4}$, \\ ISRAEL TEOLDO ${ }^{1,2}$ \\ ${ }^{1}$ Federal University of Viçosa, Viçosa, Brazil \\ ${ }^{2}$ Centre of Research and Studies in Soccer, Viçosa, Brazil \\ ${ }^{3}$ College of Healthcare Sciences, James Cook University, Queensland, Australia \\ ${ }^{4}$ Department of Medicine and Aging Sciences, 'G. d'Annunzio' University of Chieti-Pescara, Chieti-Pescara, Italy
}

\section{ABSTRACT}

Purpose. The aim of the study was to verify the effect of mental fatigue on tactical actions quality and the intensity of covered distance in soccer players.

Methods. Six U-15 male soccer players participated in this study (14.7 \pm 0.59 years of age). For data collection, the FUTSAT and GPSports ${ }^{\circledR}$ (SPI-HPU) systems were applied. To induce mental fatigue, the Stroop task was used for 20 minutes. The individuals were evaluated in 2 conditions: mental fatigue and control (with and without the Stroop task). Statistical analysis was performed with the Kolmogorov-Smirnov test, $\mathrm{t}$-test, and Wilcoxon test.

Results. The results displayed significant differences in actions related to principles of balance $(p=0.02)$, defensive unity $(p<0.01)$, and total defensive actions $(p=0.02)$, as well as in the covered distance in intensity related to zones $3(p=0.03)$ and $6(p<0.01)$. In mental fatigue, the players revealed a decrease in tactical action quality referring to movements that aimed at numerical stability in relations of opposition and at defending in unity and in block, and covered greater distances in zones 3 and 6.

Conclusions. Mental fatigue modified the players' movement dynamics on the pitch with reference to the quality of tactical actions and covered distance intensity.

Key words: football, cognitive fatigue, tactical principles, FUT-SAT, physical performance

\section{Introduction}

Mental fatigue is understood as the sensation experienced during or after long periods of cognitive activity, defined by fatigue and absence of energy [1,2]. During a soccer game, mental fatigue can be generated through the heightened cognitive demand of players to recognize, process, and interpret much information simultaneously, anticipate opponent actions and make decisions under frequent time pressured situations [3, 4]. Studies have shown the effects of mental fatigue are related to reduction of the capability of processing information [5], maintaining high levels of attention [6], anticipating actions [7], and making decisions [8]. Furthermore, recent studies suggest that mental fatigue promotes negative effects in endurance activities [9], physical tests, and executing technical abilities [10].

According to literature, a theory that explains the regulation of physical performance is the psychobiological model proposed by Marcora, Staiano, and Manning [2]. In this model, such psychobiological aspects as perceptive and motivational ones are of great importance in regulating performance and exercise tolerance [11]. Marcora, Staiano, and Manning revealed the first empirical evidence that fatigue derived from a mental effort could impair physical performance [2]. Their study evidenced, in a constant load cycle ergome-

Correspondence address: Caito André Kunrath, Universidade Federal de Viçosa - Departamento de Educação Física, Avenida Peter Henry Rolfs, s/n - Campus Universitário, Viçosa - MG, 36570-900, Brazil, e-mail: caitoandre@gmail.com

Received: February 04, 2018

Accepted for publication: March 25, 2018

Citation: Kunrath CA, Cardoso F, Nakamura FY, Teoldo I. Mental fatigue as a conditioner of the tactical and physical response in soccer players: a pilot study. Hum Mov. 2018;19(3):16-22; doi: https://doi.org/10.5114/hm.2018.76075. 
ter test, exhaustion in a reduced period when mental fatigue was induced compared with control conditions. No difference was found in physiological indicators such as oxygen uptake, heart rate, or lactate concentration between the control conditions and mental fatigue. Since this evidence, an increased interest in investigations that verify the effect of mental fatigue on sports performance, particularly in soccer, has been noticed [12-14].

Studies in soccer have indicated that mental fatigue impairs primarily physical and technical performance. For instance, in a study by Smith, Marcora, and Coutts [14], 10 individuals (among them, soccer players, Australian football players, and rugby players) performed a self-paced protocol with 6 different intensities (stand, walk, jog, run, fast run, and sprint) on a treadmill. Comparing the performance of the players between the control and experimental conditions, the results indicated a smaller total distance covered on the test, and also a smaller average speed at lower speed stages, like walk and jog, in mental fatigue. In another study, Smith et al. [10] investigated the effects of mental fatigue on maximal physical capabilities in recreational soccer players. When mentally fatigued, the subjects revealed a reduction of $16.3 \%$ of covered distance on the Yo-Yo intermittent recovery test, level 1.

Regarding technical variables, Smith et al. [10] identified that, beyond impairing physical performance, mental fatigue also influenced technical performance. In a technical test with kicking and passing fundamentals, the players presented a higher number of penalties by infractions on the passing test and also an inferior accuracy in kicking and ball speed while finishing on goal [10]. Badin et al. [13] verified the effects of mental fatigue in technical and physical performance in small-sided soccer games. Although the results did not display negative effect on the physical performance of the players, such as acceleration, number of sprints, and distance covered, the authors found a negative impact of mental fatigue on technical actions as passing and tackling. More recently, Coutinho et al. [15] observed mental fatigue effects on indicators of collective behaviours in small-sided games. Mental fatigue caused decrements in the team's synchronization behaviours such as lateral synchronization, dispersion, and contraction speed. These papers demonstrate that understanding the effects of mental fatigue is considered a challenge for researchers in soccer [10,12].

Despite the indications of negative effects of mental fatigue in physical, technical, cognitive, and collective behaviour performance in soccer $[8,10,14,15]$, investigation is necessary to identify possible influence of mental fatigue on tactical performance. Hence, interaction of tactical and physical aspects in this condition is also unknown. Considering the core tactical principles of soccer will provide information about the tactical performance embraced by players in these conditions. Therefore, this study aims to verify the effect of mental fatigue on the quality of tactical actions and in the intensity of covered distance in soccer players.

On the basis of the indicated evidence $[8,10,13]$, the hypothesis of this study is that mental fatigue causes a decrease in tactical and physical performance of soccer players. Given the importance and difficulty of managing mental fatigue and its effect in soccer, investigating the consequences of this phenomenon will allow a better understanding of potential factors that influence sports performance [16].

\section{Material and methods}

\section{Participants}

The sample comprised 6 male soccer players (14.7 \pm 0.59 years of age) who performed the total of 421 tactical actions. The subjects participated in regional and state championships, playing for a U-15 soccer team. The athlete and team selection, based on tactical, physical, and technical levels [17], was carried out by the teams' head coach. As an inclusion criterion, all players must have participated in systemized training, with at least 3 training sessions per week, each lasting 1,5-hour.

\section{Experimental protocol}

Tactical and physical actions were evaluated in 2 separate periods, with an interval of 7 days between the assessments. In the first, control condition, the players participated in a soccer field test lasting 12 minutes, with a configuration of 'goalkeeper +3 vs. $3+$ goalkeeper.' In the second, mental fatigue condition, the subjects had to perform a cognitive task for 20 minutes [18] before realizing the 'goalkeeper +3 vs. $3+$ goalkeeper' that lasted 12 minutes. The interval between the 'goalkeeper +3 vs. $3+$ goalkeeper' and the Stroop task was approximately 40 seconds, this time being devoted to walking form the place of the Stroop task to the field. To avoid cognitive recovery, no warmup was performed before the soccer field test.

In order to avoid any potential influences on game performance, the participants were not allowed to play any official soccer game for 48 hours before data collection. Moreover, they were advised not to realize any 
type of vigorous physical exercise for 24 hours before the intervention, as well as to sleep 7 hours during the previous night. The players were also instructed not to consume any drinks containing alcohol or caffeine between the last meal (lunch) and the data collection. Field sessions started between 16:00 and 17:00, as the players took part in the training sessions of the team. The average temperature on the first day of data collection was $25.8^{\circ} \mathrm{C}$ with $62 \%$ relative humidity, and on the second day $19.6^{\circ} \mathrm{C}$ with $78 \%$ relative humidity.

\section{Quality in tactical actions}

The System of Tactical Assessment in Soccer (FUTSAT) was used to collect data regarding the quality of the players' tactical actions [19]. FUT-SAT provides information on tactical behaviour in game situations on the basis of soccer core tactical principles. This evaluation considers 10 core tactical principles in soccer: 5 of the offensive phase (penetration, offensive coverage, width and length, depth mobility, offensive unity) and 5 of the defensive phase (delay, defensive coverage, balance, concentration, defensive unity).

FUT-SAT comprises 2 macro-categories. The observation macro-category refers to variables based on tactical principles, the location of the action in the pitch, and the action result. The product macro-category is related to the tactical performance index (TPI), tactical actions, positive action percentage, and location of relative action to the principles. FUT-SAT utilized to describe the quality of tactical actions presents the percentage of positive actions performed by the players.

The FUT-SAT test was structured in a 36-meter long and 27-meter wide field (Figure 1), and conducted in 3 consecutive periods of 4 minutes, with the total duration of 12 minutes. While accomplishing the test, the subjects were divided into 2 teams, each with 3 outfield players (defender, midfielder, and attacker) and a goalkeeper (goalkeeper +3 vs. $3+$ goalkeeper). Each team had numbered vests of a unique colour. The participants were requested to play by the official game rules during the test. A period of 30 seconds for familiarization with the test was conceded.

To analyse the FUT-SAT field test data, procedures proposed by Teoldo et al. [19] were followed. After recording the test, the obtained video material was introduced, in a digital format, to a portable computer (DELL model Inspiron N4030, Intel Core ${ }^{\mathrm{TM}} \mathrm{i} 3$ processor) via a USB cable and converted to an AVI file with the Format Factory video converter. For video management and analysis, the Soccer Analyzer ${ }^{\circledR}$ software was used. Match analysis was performed by a trained

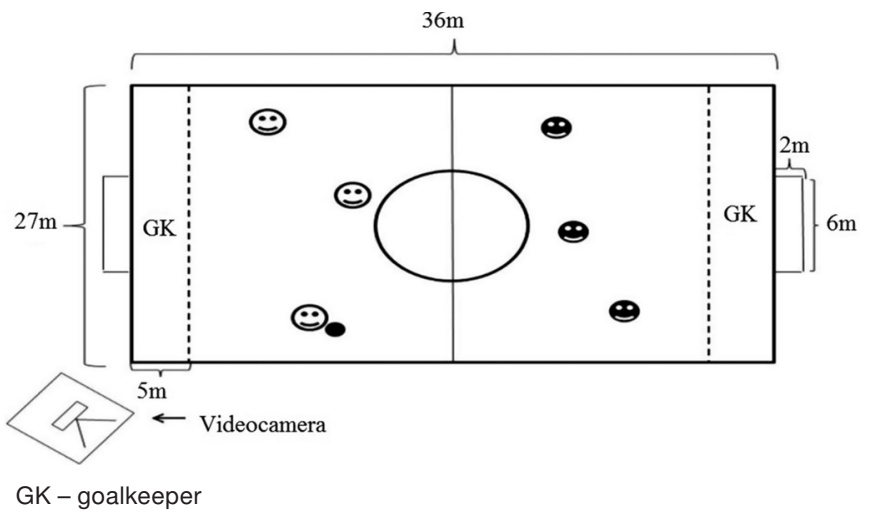

Figure 1. Representation of the FUT-SAT field test physical structure

evaluator and subjected to the procedure and methods of the training process with the FUT-SAT.

\section{Covered distance monitoring}

During the soccer field test, 6 units of a global tracking device (SPI-HPU - GPSports ${ }^{\circledR}$, Canberra) with an integrated triaxial accelerometer were used for data collection. Sampling frequency for the GPS was $15 \mathrm{~Hz}$, whereas the integrated accelerometer was defined at $100 \mathrm{~Hz}$. Once activated, the GPS unit calculated the precise distance covered on the basis of the receipt of satellite time and position data. During data collection, reception from at least 4 satellites was maintained to ensure acceptable accuracy, and the sky was clean. The intensity zones of the covered distance used in the study were: zone $1(0-6.9 \mathrm{~km} / \mathrm{h})$, zone $2(7-9.9 \mathrm{~km} / \mathrm{h})$, zone $3(10-12.9 \mathrm{~km} / \mathrm{h})$, zone $4(13-15.9 \mathrm{~km} / \mathrm{h})$, zone 5 $(16-17.9 \mathrm{~km} / \mathrm{h})$, and zone $6(\geq 18 \mathrm{~km} / \mathrm{h})$, as indicated by Aguiar et al. [20].

\section{Stroop task}

The purpose of the Stroop task was to induce the players' mental fatigue by incongruous stimulation [21]. They had to verbalize the colour of the word and ignore its meaning. In this task, 4 words ('red,' 'blue,' 'green,' and 'yellow') were presented individually on a computer in a random order of the red, blue, green, and yellow colours, and the evaluated subject was to answer, verbally, each stimulus within 1 second. For example, when the word 'red' was presented in green, the correct response to be verbalized was 'green.'

The task was executed in a closed environment, free from sound interference, and applied to all participants at the same moment. The players were seated in separate rooms and instructed individually by the researchers group, with standard task guidelines. In 
total, the participants were subjected to 1200 stimuli within 20 minutes.

\section{Statistical analysis}

To obtain information on the sample, descriptive analysis was attained (mean and standard deviation). The normality of data distribution was tested with the Kolmogorov-Smirnov test. For parametric data, paired $t$-test was utilized, and for non-parametric data, the Wilcoxon test was applied. In this analysis, the effect size was represented by Pearson's $r$, with the following classifications: low $(\leqslant 0.29)$, medium (0.30-0.49), and high $(\geq 0.50)$ [22], and the confidence interval adopted was $95 \%$.

For data generated by FUT-SAT, the test-retest method was used to verify the reliability coefficient between and within evaluators, in which Cohen's kappa values were applied in the results description. An intrarater reliability analysis was performed respecting the 21-day interval to avoid problems with the familiarity of the task. Out of the total of 421 tactical actions, 50 actions were analysed, representing $11.87 \%$ of the sample, amount higher than the reference (10\%) established in the literature. The level of reliability found was $0.818(e p=0.054)$, situated among the 'almost perfect' values (0.81-1), which demonstrates a high level of intra-rater agreement. The significance level assumed was $p<0.05$. For data processing, the Statistical Package for Social Sciences (SPSS) software for Windows, version 23.0, was used.

\section{Ethical approval}

The research related to human use has been complied with all the relevant national regulations and institutional policies, including the National Health Council resolution (466/2012), has followed the tenets of the Declaration of Helsinki, and has been approved by the Research in Human Beings Ethics Committee (CAAE: 70049717.0.0000.5153).

\section{Informed consent}

Informed consent has been obtained from all individuals included in this study and their legal guardians. Data collection was performed with the consent of the team executives.

\section{Results}

Table 1 presents the data referring to the tactical action quality within the offensive and defensive principles in the control and mental fatigue conditions.

The results revealed a decrease in tactical action quality in mental fatigue conditions when compared with the control conditions in the 'balance' defensive tactical principle $\left(\mathrm{z}_{(6)}=-2.21 ; p=0.02 ; r=0.72\right)$, 'defensive unity' $(t=4.20 ; p<0.01 ; r=0.88)$, and 'defensive total' $\left(\mathrm{z}_{(6)}=-2.20 ; p=0.02 ; r=0.78\right)$.

Table 2 results present an increase in the distance covered in zone $3(t=-2.96 ; p=0.03 ; r=0.79)$ and in zone $6(t=-5.87 ; p<0.01 ; r=0.93)$ in mental fatigue conditions when compared with the control conditions. Although the difference was not significant, it is worth

Table 1. Tactical action quality: means and standard deviations

\begin{tabular}{ccc}
\hline Control & Mental fatigue & $p$ \\
\hline success (\%) & success (\%) & \\
\hline
\end{tabular}

\begin{tabular}{lccc}
\hline Offensive principles & & & \\
Penetration & $88.85 \pm 13.21$ & $91.56 \pm 05.15$ & 0.46 \\
Offensive coverage & $99.43 \pm 01.41$ & $97.17 \pm 04.09$ & 0.28 \\
Depth mobility & $80.00 \pm 24.49$ & $88.89 \pm 27.22$ & 1.00 \\
Width and length & $97.76 \pm 01.88$ & $93.82 \pm 08.47$ & 0.22 \\
Offensive unity & $87.90 \pm 07.08$ & $92.87 \pm 05.37$ & 0.34 \\
Offensive total & $94.43 \pm 02.80$ & $93.53 \pm 04.30$ & 0.11 \\
\hline Defensive principles & & & 0.14 \\
Delay & $71.83 \pm 12.85$ & $83.30 \pm 10.88$ \\
Defensive coverage & $95.00 \pm 08.37$ & $77.62 \pm 22.19$ \\
Balance & $91.57 \pm 06.57$ & $66.65 \pm 12.46$ & $0.02^{*}$ \\
Concentration & $95.48 \pm 08.10$ & $92.41 \pm 12.92$ & 0.71 \\
Defensive unity & $88.45 \pm 03.94$ & $73.35 \pm 08.93$ & \multicolumn{2}{c}{$0.01^{*}$} \\
Defensive total & $88.52 \pm 02.72$ & $75.87 \pm 06.58$ & $0.02^{*}$ \\
\hline
\end{tabular}

* significant difference of $p<0.05$; the confidence interval adopted was $95 \%$ 
Table 2. Physical performance: means and standard deviations

\begin{tabular}{lrrc}
\hline Covered distance $(\mathrm{m})$ & \multicolumn{1}{c}{ Control } & \multicolumn{1}{c}{ Mental fatigue } & $p$ \\
\hline Total distance & $1316.83 \pm 94.93$ & $1398.02 \pm 77.64$ & 0.16 \\
Zone $1(0-6.9 \mathrm{~km} / \mathrm{h})$ & $845.83 \pm 62.58$ & $811.09 \pm 38.21$ & 0.13 \\
Zone $2(7-9.9 \mathrm{~km} / \mathrm{h})$ & $172.33 \pm 37.20$ & $190.50 \pm 28.60$ & 0.43 \\
Zone $3(10-12.9 \mathrm{~km} / \mathrm{h})$ & $69.16 \pm 11.09$ & $81.67 \pm 10.05$ & $0.03^{*}$ \\
Zone $4(13-15.9 \mathrm{~km} / \mathrm{h})$ & $6.16 \pm 03.49$ & $6.17 \pm 02.79$ & 1.00 \\
Zone $5(16-17.9 \mathrm{~km} / \mathrm{h})$ & $55.50 \pm 17.66$ & $65.17 \pm 14.02$ & 0.19 \\
Zone $6(\geq 18 \mathrm{~km} / \mathrm{h})$ & $167.33 \pm 41.16$ & $242.66 \pm 56.07$ & $<0.01^{*}$ \\
\hline
\end{tabular}

* significant difference of $p<0.05$

asserting that in mental fatigue, the players also covered greater total distances with lower speeds.

\section{Discussion}

The study aimed to verify the effects of mental fatigue in tactical actions quality and in the distance covered by soccer players in different intensities. The results indicated a decrease in action quality related to the defensive tactical principles of balance, defensive unity, and the total of defensive actions. Regarding the physical performance results, an increase of the distance covered in speeds corresponding to zone 3 $(10-12.9 \mathrm{~km} / \mathrm{h})$ and of higher intensities $(\geq 18 \mathrm{~km} / \mathrm{h})$ was observed.

The decrease in action quality referring to the tactical principles is related to the players' exposure to mental fatigue. In this context, mental fatigue could not determine the capability of individuals in realizing tasks, but influenced the difficulty in maintaining high levels of performance [12]. Regarding tactical actions, the players' exposure to mental fatigue caused a decrease in actions related to defensive principles, primarily in actions farther from the centre of play. The centre of play is the space where the game occurs with higher intensity and speed, and ball transmission among teammates is facilitated [23].

The tactical principle of balance has the purpose of assuring defensive organization by means of numbered equality or superiority of defensive players who are positioned between the ball and their own goal [24]. Poor quality of tactical actions within this principle does not offer safety to players involved in defensive actions inside the centre of play, leaving spaces uncovered, free passing lanes, and potential players to receive passes free from marking [23]. Yet, the defensive unity principle has strong connections with game understanding and player positioning organization [24]. This principle aims to ensure guiding lines that coordinate the players' behaviour inside the centre of play.
An unskilled implementation of these actions does not provide the necessary organization so defensive players press the opposing team, allowing bigger spaces for the opposing players' offensive organization [21].

Regarding the effects of mental fatigue on the possible indicators of tactical performance, a recent study by Coutinho et al. [15] investigated the effects of mental fatigue on players' capability to coordinate team movements. The authors induced mental fatigue by a motor coordination task and observed some decrements on the team's behavioural synchronization: lateral synchronization, dispersion, and contraction speed. Although this paper presented only indicators of collective behaviours, the results can be linked with the present study and provide an interesting insight to prospective studies in this area.

The results of the present study also revealed that players, when exposed to a mental fatigue condition, cover greater distances in speed corresponding to zones 3 and $6(10-12.9 \mathrm{~km} / \mathrm{h}$ and $\geq 18 \mathrm{~km} / \mathrm{h})$. Thus, it is possible to speculate that the increase of the covered distance in this intensity was a consequence of the decrease of action quality related to the defensive tactical principle. This happens because actions related to the defensive principles of balance and defensive unity are executed further away from the ball, demanding players' approximation to the centre of play to enable adequate intervention. Consequently, it is understood that the increase in the covered distance in speed corresponding to zones 3 and 6 was a strategy assumed by the players, looking to compensate for the decrease in tactical action quality.

Relating to this strategy, Badin et al. [13] specify that small-sided games allow adjustments in game rhythm, as players look to balance the performance on the basis of different game situations. These authors also do not suggest a negative effect of mental fatigue on physical performance, however, in this condition, a decrease was noted in the quality of technical actions of passing, tackling, and dribbling. It is worth pointing out that 
the present study does not counter previous results in literature $[10,14]$ reporting that mental fatigue causes negative effects on endurance tests with constant load or in physical tests because, during a game, players are free to alter their pace and are not demanded maximum exercise.

Other studies in soccer indicate results opposite to this study, verifying that mental fatigue impairs physical performance. Negative effect on performance was found in intermittent running in physical treadmill tests, and a decrease of $16.3 \%$ of the covered distance was observed in the Yo-Yo intermittent recovery test, level 1. However, results from the use of physical tests with the purpose of verifying the effects of mental fatigue in physical performance should not be necessarily transferred to the game because they do not approach game situations environment. Badin et al. [13] displayed rules that modify the main objective of the game, such as 6 consecutive passes among players of a team to obtain one 'point' and absence of a goalkeeper. Moreover, their study prioritized conserving the internal logic and the official game rules, with the assumption that this would make the results a better illustration of the formal game conditions.

The hypothesis that mental fatigue caused negative effects on tactical action quality and intensity in the distance covered by players was confirmed. Studies show that mental fatigue impairs physical performance in such sports as running [25], cycling [26], and cricket [27], as well as in soccer physical tests [10, 14]. Although the results indicated an increase in the distance covered in speeds corresponding to zone 3 $(10-12.9 \mathrm{~km} / \mathrm{h})$ and in higher intensity zones $(\geq 18 \mathrm{~km} / \mathrm{h})$, we understand that covering greater distances in elevated speeds as a consequence of poor tactical actions can negatively influence soccer players' performance.

In terms of practical implications, mental fatigue should be considered as a potential factor modifying tactical and physical actions in a soccer game. Beside the negative effects on tactical performance, mental fatigue almost induces players to cover greater distances with elevated speeds, which can cause greater difficulty in the recovery process during matches and in intervals between training and games [28], thus increasing the risk of muscle injury in players [29]. This information attracts attention to the importance of considering mental fatigue as a relevant variable in soccer.

The study limitations include the lack of data regarding the control of individual performance with respect to psychological indicators, as well the maturational status of the players. These data could pro- vide additional information to help understand the behaviour displayed by the participants. Moreover, future research should replicate this experimental protocol with a greater sample to confirm the results. It is also necessary to consider individual thresholds of mental fatigue by controlling mental fatigue tasks. Thus, further studies should investigate associations among cognitive efficiency and impairments in tactical, technical, and physical performance in a soccer game.

\section{Conclusions}

The results suggest that mental fatigue impairs players' performance, inducing them to apply poorly qualified defensive tactical actions and to cover greater distances in speeds corresponding to zones 3 (10$12.9 \mathrm{~km} / \mathrm{h})$ and $6(\geqslant 18 \mathrm{~km} / \mathrm{h})$. Furthermore, it is concluded that mental fatigue alters the players' movement dynamics in the field, which refers to tactical action quality and covered distance intensity.

\section{Acknowledgments}

The study was supported by the State Department of Sport and Youth of Minas Gerais (SEESP-MG) in accordance with the State Act of Incentive to Sports, by FAPEMIG, CAPES, CNPQ, FUNARBE, the Dean's Office for Graduate and Research Studies, and the Centre of Life and Health Science at the Federal University of Viçosa, Brazil.

\section{Disclosure statement}

No author has any financial interest or received any financial benefit from this research.

\section{Conflict of interest}

The authors state no conflict of interest.

\section{References}

1. Boksem MA, Tops M. Mental fatigue: costs and benefits. Brain Res Rev. 2008;59(1):125-139; doi: 10.1016/j. brainresrev.2008.07.001.

2. Marcora SM, Staiano W, Manning V. Mental fatigue impairs physical performance in humans. J Appl Physiol. 2009;106(3):857-864; doi: 10.1152/japplphysiol.91324. 2008.

3. Nédélec M, McCall A, Carling C, Legall F, Berthoin S, Dupont G. Recovery in soccer: part I - post-match fatigue and time course of recovery. Sports Med. 2012;42(12): 997-1015; doi: 10.2165/11635270-000000000-00000.

4. Williams AM, Reilly T. Talent identification and development in soccer. J Sports Sci. 2000;18(9):657-667; doi: 10.1080/02640410050120041. 
5. Lorist MM, Klein M, Nieuwenhuis S, De Jong R, Mulder G, Meijman TF. Mental fatigue and task control: planning and preparation. Psychophysiology. 2000;37(5): 614-625; doi: 10.1111/1469-8986.3750614.

6. Boksem MA, Meijman TF, Lorist MM. Effects of mental fatigue on attention: an ERP study. Brain Res Cogn Brain Res. 2005;25(1):107-116; doi: 10.1016/j.cogbrainres.2005.04.011.

7. Duncan MJ, Fowler N, George O, Joyce S, Hankey J. Mental fatigue negatively influences manual dexterity and anticipation timing but not repeated high-intensity exercise performance in trained adults. Res Sports Med. 2015;23(1):1-13; doi: 10.1080/15438627.2014.975811.

8. Smith MR, Zeuwts L, Lenoir M, Hens N, De Jong LMS, Coutts AJ. Mental fatigue impairs soccer-specific decision-making skill. J Sports Sci. 2016;34(14):1297-1304; doi: 10.1080/02640414.2016.1156241.

9. Van Cutsem J, Marcora S, De Pauw K, Bailey S, Meeusen R, Roelands B. The effects of mental fatigue on physical performance: a systematic review. Sports Med. 2017; 47(8):1569-1588; doi: 10.1007/s40279-016-0672-0.

10. Smith MR, Coutts AJ, Merlini M, Deprez D, Lenoir M, Marcora SM. Mental fatigue impairs soccer-specific physical and technical performance. Med Sci Sports Exerc. 2016;48(2):267-276; doi: 10.1249/MSS.00000 00000000762.

11. Pageaux B. The psychobiological model of endurance performance: an effort-based decision-making theory to explain self-paced endurance performance. Sports Med. 2014;44(9):1319-1320; doi: 10.1007/s40279014-0198-2.

12. Cárdenas D, Conde-González J, Perales JC. The role of mental workload in sport training planning [in Spanish]. Rev Psicol del Deport. 2015;24(1):91-100.

13. Badin OO, Smith MR, Conte D, Coutts AJ. Mental fatigue impairs technical performance in small-sided soccer games. Int J Sports Physiol Perform. 2016;11(8): 1100-1105; doi: 10.1123/ijspp.2015-0710.

14. Smith MR, Marcora SM, Coutts AJ. Mental fatigue impairs intermittent running performance. Med Sci Sports Exerc. 2015;47(8):1682-1690; doi: 10.1249/ MSS.0000000000000592.

15. Coutinho D, Gonçalves B, Travassos B, Wong DP, Coutts AJ, Sampaio JE. Mental fatigue and spatial references impair soccer players' physical and tactical performances. Front Psychol. 2017;8:1645; doi: 10.3389/ fpsyg.2017.01645.

16. Coutts AJ. Fatigue in football: it's not a brainless task! J Sports Sci. 2016;34(14):1296; doi: 10.1080/02640414. 2016.1170475.
17. Casamichana D, Castellano J. Time-motion, heart rate, perceptual and motor behaviour demands in small-sides soccer games: effects of pitch size. J Sports Sci. 2010; 28(14):1615-1623; doi: 10.1080/02640414.2010.521168.

18. Nuechterlein KH, Parasuraman R, Jiang Q. Visual sustained attention: image degradation produces rapid sensitivity decrementovertime. Science. 1983;220(4594): 327-329; doi: 10.1126/science.6836276.

19. Teoldo I, Garganta J, Greco PJ, Mesquita I. Proposal for tactical assessment of soccer player's behaviour regarding core principles of the game [in Portuguese]. Motriz. 2011;17(3):511-524; doi: 10.1590/S1980-65742011 000300014.

20. Aguiar MV, Botelho GM, Gonçalves BS, Sampaio JE. Physiological responses and activity profiles of soccer small-sided games. J Strength Cond Res. 2013;27(5): 1287-1294; doi: 10.1519/JSC.0b013e318267a35c.

21. Pageaux B, Lepers R, Dietz KC, Marcora SM. Response inhibition impairs subsequent self-paced endurance performance. Eur J Appl Physiol. 2014;114(5):10951105; doi: 10.1007/s00421-014-2838-5.

22. Cohen J. Statistical power analysis for the behavioral sciences. New York: Lawrence Erlbaum Associates; 1988.

23. Teoldo I, Guilherme J, Garganta J. Training football for smart playing: on tactical performance of teams and players. Curitiba: Appris; 2017.

24. Teoldo I, Garganta JM, Greco PJ, Mesquita I. Tactical principles of a soccer game: concepts and application [in Portuguese]. Motriz. 2009;15(3):657-668.

25. MacMahon C, Schücker L, Hagemann N, Strauss B. Cognitive fatigue effects on physical performance during running. J Sport Exerc Psychol. 2014;36(4):375381; doi: 10.1123/jsep.2013-0249.

26. Martin K, Staiano W, Menaspà P, Hennessey T, Marcora S, Keegan R, et al. Superior inhibitory control and resistance to mental fatigue in professional road cyclists. PLoS One. 2016;11(7):e0159907; doi: 10.1371/ journal.pone.0159907.

27. Veness D, Patterson SD, Jeffries O, Waldron M. The effects of mental fatigue on cricket-relevant performance among elite players. J Sports Sci. 2017;35(24):24612467; doi: 10.1080/02640414.2016.1273540.

28. Reilly T, Ekblom B. The use of recovery methods postexercise. J Sports Sci. 2005;23(6):619-627; doi: 10.1080/ 02640410400021302.

29. Fatouros IG, Chatzinikolaou A, Douroudos II, Nikolaidis M, Kyparos A, Margonis K, et al. Time-course of changes in oxidative stress and antioxidant status responses following a soccer game. J Strength Cond Res. 2010;24(12):3278-3286; doi: 10.1519/JSC.0b013e3181 b60444. 